\title{
Language-Related Activations in the Left Prefrontal Regions Are Differentially Modulated by Age, Proficiency, and Task Demands
}

\author{
Yoshinori Tatsuno ${ }^{1,2}$ and Kuniyoshi L. Sakai ${ }^{1,2}$ \\ ${ }^{1}$ Department of Basic Science, Graduate School of Arts and Sciences, The University of Tokyo, Komaba, Tokyo 153-8902, Japan, and ${ }^{2}$ Core Research for \\ Evolutional Science and Technology, Japan Science and Technology Agency, Kawaguchi-shi 332-0012, Japan
}

It remains to be elucidated how cortical activations are modulated by factors of age, proficiency, and language task demands when mastering first language (L1) and a second language (L2). Using functional magnetic resonance imaging, we tested subjects aged 13 (the age 13 group) and 19 (the age 19 group), thereby comparing the cortical activations involved in past-tense verb identification with those involved in verb matching. We found that the activation in the dorsal triangular part of the left inferior frontal gyrus (IFG) was lower, corresponding to a higher proficiency in English (L2) in the older subjects, suggesting that the proficiency level plays a major role in the activation of this region during L2 acquisition. Moreover, the lower activation in the triangular and orbital parts of the left IFG (F3t/F30) for the irregular past tense corresponding to a higher proficiency in L2, together with the nonsignificant activation for the regular past tense when its performance almost reached perfection for age 19, suggests that the modulation of the left F3t/F30 activation reflects language task demands for identifying correct past-tense forms. On the other hand, the left F3t/F30 activation in Japanese (L1) for age 13 was significantly greater than that for age 19, despite the matched performances in L1. These results suggest that the left IFG subserves language-specific functions that are critically required when mastering any language.

Key words: language acquisition; grammar; prefrontal cortex; fMRI; cortical plasticity; critical period

\section{Introduction}

The developmental processes in the brain for mastering first language (L1) and a second language (L2) are essentially unknown, and the question of whether they share the same neural mechanisms remains controversial. Given the fact that the proficiency level (PL) of L2 rarely becomes comparable with that of L1 if L2 is acquired beyond the hypothesized sensitive period around puberty (Lenneberg, 1967; Johnson and Newport, 1989), the age of acquisition (AOA) is regarded as one critical factor. It has been reported that L1 (AOA, before $\sim 6$ years) and L2 (AOA, after $\sim 7$ years) are represented differentially in cortical areas during discourse production or passive listening tasks (Dehaene et al., 1997; Kim et al., 1997), whereas other studies have reported that they have common neural substrates during passive listening or word generation tasks (Perani et al., 1998; Chee et al., 1999). A recent functional magnetic resonance imaging (fMRI) study supports the AOA effect on cortical activations, such that the left inferior frontal gyrus (IFG) activation for grammatical processing in L2 is

\footnotetext{
Received May 13, 2004; revised Nov. 19, 2004; accepted Nov. 27, 2004.

This work was supported by a Core Research for Evolutional Science and Technology grant from the Japan Science and Technology Agency and by a Young Investigators' Grant from the Human Frontier Science Program (K.L.S.). We thank Y. Muraishi, K. Miura, N. Narafu, T. Mitsuhashi, K. Hirano, M. Fukushima, Y. Tanabe, A. Katsumata, and Y. Sugiura for their help in studying school students and N. Saeki for technical assistance.

Correspondence should be addressed to Dr. Kuniyoshi L. Sakai, Department of Basic Science, Graduate School of Arts and Sciences, The University of Tokyo, Komaba, 3-8-1 Komaba, Meguro-ku, Tokyo 153-8902, Japan. E-mail: sakai@mind.c.u-tokyo.ac.jp.

D0I:10.1523/JNEUROSCI.3978-04.2005

Copyright $\odot 2005$ Society for Neuroscience $\quad 0270-6474 / 05 / 251637-08 \$ 15.00 / 0$
}

greater than that in L1 (Wartenburger et al., 2003). However, another fMRI study claims that the degree of exposure to language affects the left IFG activation, even if the AOA is matched (Perani et al., 2003). Indeed, the PL effect is known to modulate the left IFG activation in adults during semantic or grammatical decision tasks (Chee et al., 2001; Musso et al., 2003), as well as the bilateral temporal lobe activation while listening to stories (Perani et al., 1998). It has also been pointed out that the left frontal and extrastriate regions were differentially modulated by either age or task performance among children (aged 7-10) and adults (Schlaggar et al., 2002). However, the age and PL effects on cortical activations are often confounded with the demands required in each language task and the resultant task performance, and it remains unknown whether these factors are actually separable from each other.

Given these uncertainties, the purpose of the present study was to clarify the relative contributions of age, PL, language task demands, and task performance to modulating activations in the left prefrontal cortex as the subjects performed language tasks. In our previous study, we examined whether training in the conjugation of English (L2) verbs from the present to past tense altered brain activation in 13-year-old native Japanese (L1) speakers (Sakai et al., 2004). A past-tense verb identification task in English was contrasted with a verb-matching task; the former required linguistic knowledge, and the latter did not. We found that the left IFG exhibited significant activation increases for the pasttense task and that these were positively correlated with individ- 
ual improvements in PL. In the present study, by using the same paradigm, we tested native Japanese speakers aged 13 and 19 to clarify how the language task-selective activations in the left IFG are modulated by the above-mentioned factors. Here, we predict that $\mathrm{L} 2$ acquisition involves not only general learning skills but specific language mechanisms that are represented in distinct prefrontal regions.

\section{Materials and Methods}

Subjects. The subjects in the present study were 29 right-handed native Japanese speakers in two age groups: age 13 ( 13 years, $0-8$ months) and age 19 (18 years, 8 months to 19 years, 8 months). All of the subjects had studied English only in Japan, and the age at which they had begun acquiring English was 12 ( 12 years, 3 months to 13 years, 0 month). Fourteen first-year students (eight females and six males) from a secondary education school were in the age 13 group. These subjects had studied English for 8 months at school. Before the experiment, the students in this group had participated in 2 months of classroom training in the English past tense, as described in our previous study (Sakai et al., 2004). Fifteen undergraduate students (seven females and eight males) from The University of Tokyo were in the age 19 group. These subjects had studied English for 6 years only at school, through which they had learned the English past tense. Informed consent was obtained from all subjects, as well as from the parents of the 13-year-old subjects. The study was approved by the Secondary Education School Attached to the Faculty of Education of The University of Tokyo and by the institutional review board of The University of Tokyo, Komaba.

Four tasks were tested in the fMRI sessions: an English verb-matching (EM) task, an English past-tense (EP) task, a Japanese verb-matching (JM) task, and a Japanese past-tense (JP) task, as described previously (Sakai et al., 2004). In the analyses for EP and EM, each of the age 13 and age 19 groups was divided into two subgroups. The age 13 group was divided into $13 \mathrm{EH}$ (the higher score subgroup in English; $n=6$ ) and 13EL (the lower score subgroup in English; $n=8$ ) at $40 \%$ accuracy of the irregular verbs presented in the EP tasks, which was the median of accuracy for age 13. The accuracy of irregular verbs was appropriate for this group division, because we reported previously that 13-year-old students showed significant improvements in accuracy at conjugating irregular verbs by the end of 2 months of training (from $22 \pm 3.9$ to $43 \pm 6.9 \%$ ) but not for regular verbs (from $79 \pm 3.9$ to $77 \pm 3.7 \%$ ) (Sakai et al., 2004). The age 19 group was divided into 19EH $(n=7)$ and 19EL $(n=8)$ at $96 \%$ accuracy of irregular verbs presented in the EP tasks, which was the median of accuracy for age 19 . To compare the age 13 and age 19 groups under the matched performance of JP, we chose subjects who committed four errors or less out of $80 \mathrm{JP}$ trials $(>95 \%)$ from age 13 as the $13 \mathrm{JH}$ subgroup (the higher score subgroup in Japanese for age $13 ; n=6$ ) and those who committed one to three errors (96-99\%) from age 19 as the 19JL subgroup (the lower score subgroup in Japanese for age 19; $n=8$ ).

Stimuli and tasks. For the fMRI sessions, we used 48 English verbs (24 regular and 24 irregular), as well as 48 Japanese verbs that were presented in both the "hiragana" and "kanji" writing systems, and were matched with English verbs of the same meaning. The stimulus presentation and behavioral data collection were controlled using the LabVIEW software and interface (National Instruments, Austin, TX). The subjects wore earplugs and an eyeglass-like MRI-compatible display (resolution, $800 \times$ 600) (VisuaStim XGA; Resonance Technology, Northridge, CA).

In each $3 \mathrm{~s}$ trial of the fMRI experiments, word or pseudoword stimuli in yellow letters against a dark background were visually presented. For fixation, a red cross was always shown at the center of the screen. First, the letter "E" (EM), an "E" with a circle around it (EP), a "J" (JM), or a "J" with a circle around it (JP) was presented for $400 \mathrm{~ms}$ at the initiation of every trial, indicating the task to be performed. Next, a present-tense verb was shown for $1300 \mathrm{~ms}$. The subjects covertly read the presented verb for $\mathrm{EM}$ and JM or covertly read its past-tense form for EP and JP if they knew the past-tense form (if not, they read the presented verb). Finally, two choice stimuli were shown for $1000 \mathrm{~ms}$, and the subjects attempted to choose the same present-tense verb for EM and JM or attempted to choose the correct past-tense form for EP and JP, even if the verb was unfamiliar to them, by pushing one of two buttons. The time limit given for responses was as short as $1000 \mathrm{~ms}$, so that responses with preplanning during the preceding period became necessary. No feedback on performance was given to any subject. General cognitive factors such as word recognition and response selection were controlled by the EM and JM tasks, which were directly compared with the EP and JP tasks, respectively.

The four tasks were conducted in a block design; six trials were tested in each EM block, and eight trials were tested in each block for the other tasks. There were two EP task blocks in a single fMRI run: one block (EPi) with seven irregular verbs and one regular verb (at a randomized position), the other (EPr) with seven regular verbs and one irregular verb. One EM block and one block each of JM, EPi, EPr, and JP were alternately presented (a total of nine blocks) for each run. Because the main goal of the present study was to clarify the cortical activations for the English past tense, i.e., EPi-EM and EPr-EM, the alternating EM blocks served as the steady referential baseline. Ten fMRI runs were performed in a single day, and the order of tasks was counterbalanced across subjects.

fMRI data acquisition and analyses. The fMRI scans were conducted using a 1.5 T MRI system (STRATIS II, Premium; Hitachi Medical Corporation, Tokyo, Japan). Using a gradient-echo echo-planar imaging sequence (repetition time, $3 \mathrm{~s}$; echo time, $50.5 \mathrm{~ms}$; flip angle, $90^{\circ}$; field of view, $192 \times 192 \mathrm{~mm}^{2}$; resolution, $3 \times 3 \mathrm{~mm}^{2}$ ), we scanned 16 horizontal slices, each $6 \mathrm{~mm}$ thick and having a $1 \mathrm{~mm}$ gap, covering from $z=-49$ to $62 \mathrm{~mm}$. We performed group analyses using SPM99 statistical parametric mapping software (Wellcome Department of Imaging Neuroscience, London, UK). We realigned the functional volume data in multiple runs and removed runs that included data with a translation of $>2$ $\mathrm{mm}$ in one of the three directions and a rotation of $>1.4^{\circ}$. The data were normalized to the standard brain, resampled every $3 \mathrm{~mm}$ using bilinear interpolation, and smoothed with an isotropic Gaussian kernel of $9 \mathrm{~mm}$ full-width at half-maximum. Low-frequency noise and global changes in activity were further removed. Task-specific effects were estimated with a general linear model (fixed-effects analyses) using a boxcar waveform convolved with the canonical hemodynamic response function. In group analyses, statistical parametric maps in each comparison were thresholded at the corrected $p<0.05$ for the voxel level. Statistical significance of negative correlation between the accuracy of irregular verbs in the EP tasks and the signal change in either EPi-EM or EPr-EM was tested using Spearman rank correlation (one-sided), which is resistant to outliers, i.e., high leverage points.

\section{Results}

\section{Behavioral results}

We first examined the in-scanner accuracy and reaction times of the EPi and EPr tasks for the individual subject groups. The accuracy of EPi was higher for the older subjects, and the differences among the 13EL, 13EH, 19EL, and 19EH subgroups were all significant ( $t$ test; $p<0.01$ ) (Fig. $1 A$ ). The mean reaction times of EPi for 19EH were also significantly faster than those for 19EL $(p<0.05)$, indicating that there were true performance differences of EPi between 19EL and 19EH, even with an accuracy of $>90 \%$. On the other hand, the accuracy of EPr for 19EL was significantly higher than that for 13EH $(p<0.0001)$ (Fig. $1 B$ ), whereas the difference in accuracy and reaction times between $19 \mathrm{EL}$ and 19EH was not significant $(p>0.1)$, suggesting that the performance of EPr almost reached perfection for the age 19 groups. In addition, the accuracy of EPr for 13EL was significantly higher than that for $13 \mathrm{EH}(p<0.001)$, which indicated the higher probability of choosing verbs ending in "-ed." Nevertheless, the accuracy and reaction times of all irregular verbs of the EP tasks, i.e., seven verbs in a single EPi block and one verb in a single EPr block for each run, showed the same pattern as EPi (Fig. 1C). Taking both the accuracy and reaction times into account, the accuracy of irregular verbs in the EP tasks is a reliable measure of language attainment in the four subgroups of subjects. 

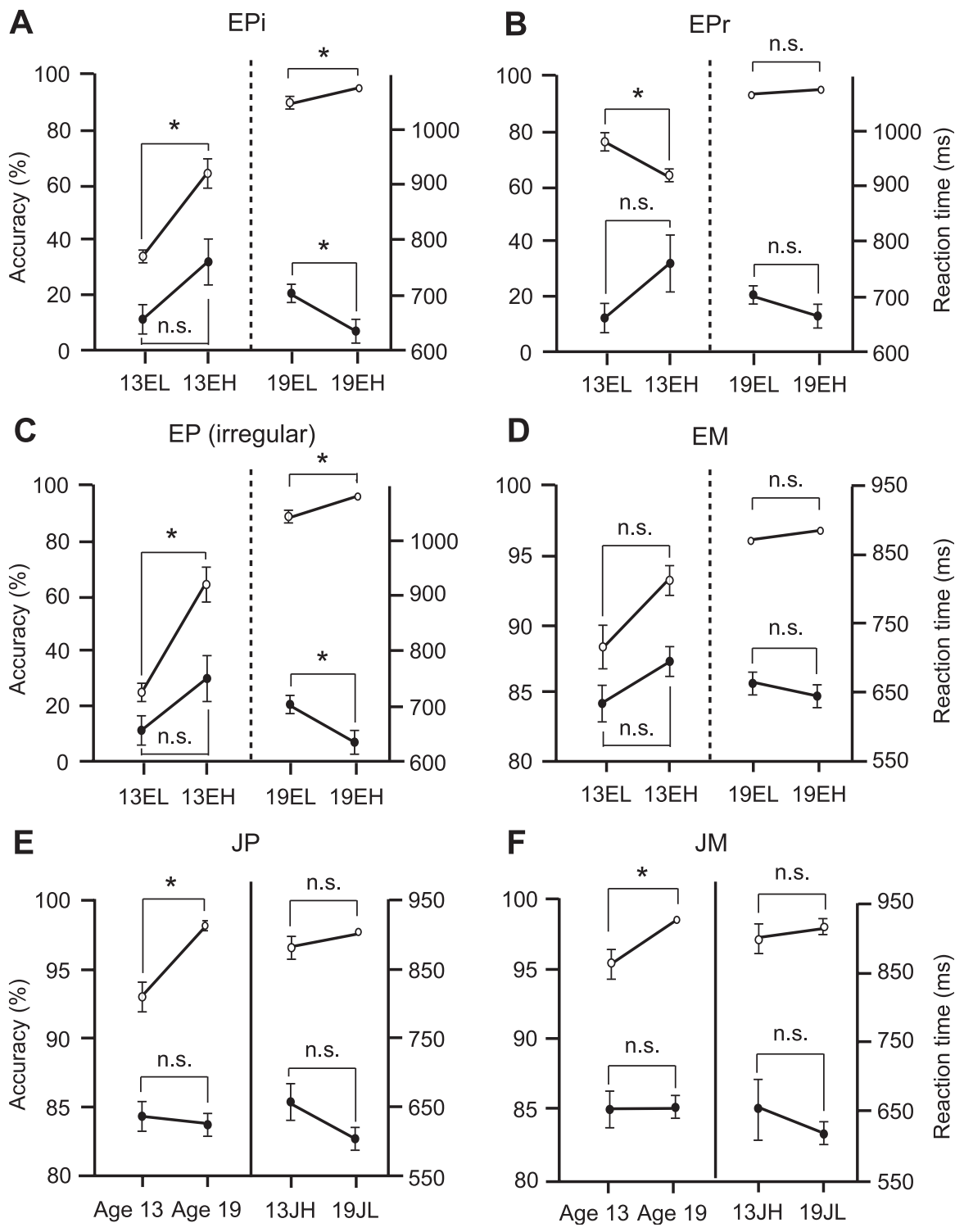

Figure 1. Accuracy and reaction times of past-tense and verb-matching tasks in English (L2) and Japanese (L1). Four tasks were tested in the fMRI sessions: an English verb-matching (EM) task, an English past-tense (EP) task, a Japanese verb-matching (JM) task, and a Japanese past-tense (JP) task. There were two EP task blocks: one block (EPi) with seven irregular verbs and one regular verb, and the other (EPr) with seven regular verbs and one irregular verb. $A-D$, The accuracy and reaction times (mean \pm SEM of the subjects) of $\operatorname{EPi}(A), \operatorname{EPr}(B)$, irregular verbs of the EP tasks $(C)$, and $E M(D)$ are shown for each of the four subgroups: 13EL (the lower score subgroup in English for age 13), 13EH (the higher score subgroup in English for age 13), 19EL (the lower score subgroup in English for age 19), and 19EH (the higher score subgroup in English for age 19). Open and filled circles denote the accuracy and reaction times, respectively. $E, F$, The accuracy and reaction times of JP $(E)$ and JM $(F)$ are shown for each age group and for performance-matched 13JH (the higher score subgroup in Japanese for age 13) and 19JL (the lower score subgroup in Japanese for age 19). Note that the ordinate scale is different between $A-C$ and $D-F$. Asterisks indicate the significant difference in accuracy or reaction times between a pair of two groups ( $t$ test; $p<0.05$ ). n.S., A nonsignificant difference. The accuracy of each English task, i.e., EPi, EPr, EP (irregular), and EM, was significantly higher for 19EL than for 13EH ( $p<0.05$ ).

In contrast to the EPi and EPr tasks, the EM, JP, and JM tasks were less demanding on the subjects. In fact, the accuracy difference of EM between the subgroups within age 13 or age 19 was not significant $(p>0.05)$, and the difference in reaction times was not significant between any pairs of subgroups $(p>0.1)$ (Fig. $1 D$ ). Although the accuracy of JP was significantly different between the age 13 and age 19 groups $(p<0.0001)$ (Fig. $1 E$ ), the reaction times of JP were not significantly different between age 13 and age $19(p>0.1)$. When the subgroups of 13JH and 19JL were selected to equate the accuracy of JP $(p>0.1)$ (Fig. $1 E)$, the reaction times of JP were also matched between these subgroups
( $p>0.05)$. These subgroups also showed similar matched performances for JM $(p>0.1)($ Fig. $1 F)$.

\section{Activation of the dorsal triangular part of the left IFG in L2 selectively modulated by PL}

The contrasts of EPi-EM and EPr-EM were first examined to establish the taskselective activations for the past-tense processing in L2 (Fig. $2 A-D$ ). The overall activation patterns were similar between EPi-EM and EPr-EM, as well as between age 13 and age 19 groups; consistent activations were observed in the left IFG, spanning from the precentral gyrus (PrCG) to the opercular part and triangular part of the IFG (F3op/F3t), the right F3op/F3t, the supplementary motor area, the bilateral parieto-occipital cortex, and the bilateral cerebellum (Table 1). For age 13 , additional activation was observed in the right orbital part of the IFG and the middle frontal gyrus (F3O/F2O) in EPiEM. For age 19, the caudate nucleus and the calcarine cortex showed additional activations in both the contrasts, as well as the right insula and the left middle frontal gyrus (F2) in EPi-EM and the bilateral insula and the left middle temporal gyrus in EPr-EM (Table 1). Regarding the difference between EPi-EM and EPr-EM, the left $\mathrm{F} 3 \mathrm{t} / \mathrm{F} 3 \mathrm{O}$ activation was found in EPi-EM for both ages (Fig. 2A,C, white circles), but not in EPr-EM (Fig. 2B,D). The similar region is shown to be active in Figure $2 D$, but the activation projected onto the lateral surface was actually located in the left insula and did not extend to the lateral $\mathrm{F} 3 \mathrm{t} / \mathrm{F} 3 \mathrm{O}$.

We reported previously that the left dorsal F3t (dF3t) [Montreal Neurological Institute (MNI) coordinates $(-51,24$, 24)] showed activation increases for age 13 , positively correlated with individual PL improvements for English past-tense verbs (Sakai et al., 2004). For the subject groups in the present study, the mean signal changes in EPi-EM and EPr-EM were calculated at the same locus (Fig. 2A-D, yellow dots). We found that the mean signal change for 19EH was significantly lower compared with that for 19EL, in both EPi-EM and EPr-EM $(p<0.05)$ (Fig. $2 E, F)$. It is striking to note that the activation in $\mathrm{EPi}-\mathrm{EM}$ and in EPr-EM at the left dF3t was lower, corresponding to a higher proficiency in English (L2) in the older subjects. The direct comparison between EPi and EPr (Fig. 2G) exhibited no significant difference for any subgroups $(p>0.1)$, and none of the pairs of groups showed significant differences $(p>0.1)$. When we considered PL (i.e., the accuracy of irregular verbs in the EP tasks) as a continuous variable treating subjects as a random effect, we found a significant negative correlation between $\mathrm{PL}$ and the left $\mathrm{dF} 3 \mathrm{t}$ activation in $\mathrm{EPi}-\mathrm{EM}(r=-0.55$; Spearman 
rank correlation coefficient, $r_{s}=-0.53$; $p<0.05$ ) (Fig. $2 H$ ), as well as in EPr-EM $\left(r=-0.61 ; r_{s}=-0.44 ; p<0.05\right)$ (Fig. $2 I)$. Because EPi-EM and EPr-EM resulted in the same pattern of activations among the individuals despite the differential performances of EPi and EPr (Fig. $1 A, B)$, the modulation of the left dF3t activation cannot be explained by the task performance. Moreover, the EM task served as the control condition for the individual groups, and thus the effects of general cognitive factors on cortical activations would have been canceled out in EP-EM. Therefore, either the PL effect alone or the interaction of PL by age explains our previous observation that the left $\mathrm{dF} 3 \mathrm{t}$ activation increases at the early stage of L2 acquisition, and our present results that it is lower when a higher proficiency in L2 is attained.

Left F3t/F3O activation in L2 selectively modulated by language task demands Given the differential activation in the left F3t/F3O between EPi-EM and EPr-EM (Fig. 2A-D), we next conducted a voxelwise ANOVA for task (EPi and EPr) and age (13 and 19) for the fMRI data sets to examine any task-related or age-related differences. The main effects of task resulted in a single activated region in the left F3t/F3O for EPi-EPr (Table 2), whereas EPr-EPi resulted in no significant activation. The main effects of age were observed in the left PrCG for age 13-age 19. For age 19-age 13, the main effects of age were observed in the left F3t, the left inferior parietal gyrus, the right middle occipital gyrus, and the calcarine cortex (Table 2). The interaction of task by age showed no significant activation.

The comparison of EPi-EPr was further performed separately for the age 13 and age 19 groups. For age 13, the direct comparison EPi-EPr did not reach the significance level, although the left F3t/F3O activation was observed in EPi-EM but not in EPr-EM (Fig. 2A, B). For age 19, the significant activation was localized in the left F3t/F3O in EPi-EPr [Fig. 3A, yellow circle $(-48,21,-3)]$. In EPi-EM (Fig. 3B), the mean signal changes at this locus were significant for all subgroups $(p<0.05)$ except $19 \mathrm{EH}$, and the signal change for $19 \mathrm{EH}$ was significantly lower compared with that for 19EL $(p<0.05)$, which was similar to the modulation of the left dF3t activation (Fig. $2 E$ ). However, in EPr-EM (Fig. 3C), no subject group showed significant activation $(p>0.05)$, and none of the pairs of subgroups showed a significant difference $(p>0.05)$. We also found a significant negative correlation between PL and the left F3t/F3O activation in EPi-EM $\left(r=-0.34 ; r_{s}=-0.49 ; p<0.05\right)$ (Fig. 3D). In contrast, the absence of a significant correlation in $\operatorname{EPr}-\mathrm{EM}(r=$ $0.07 ; r_{s}=-0.007 ; p>0.5$ ) (Fig. $3 E$ ) indicated that the left F3t/ $\mathrm{F} 3 \mathrm{O}$ activation was not modulated by PL itself. Moreover, the
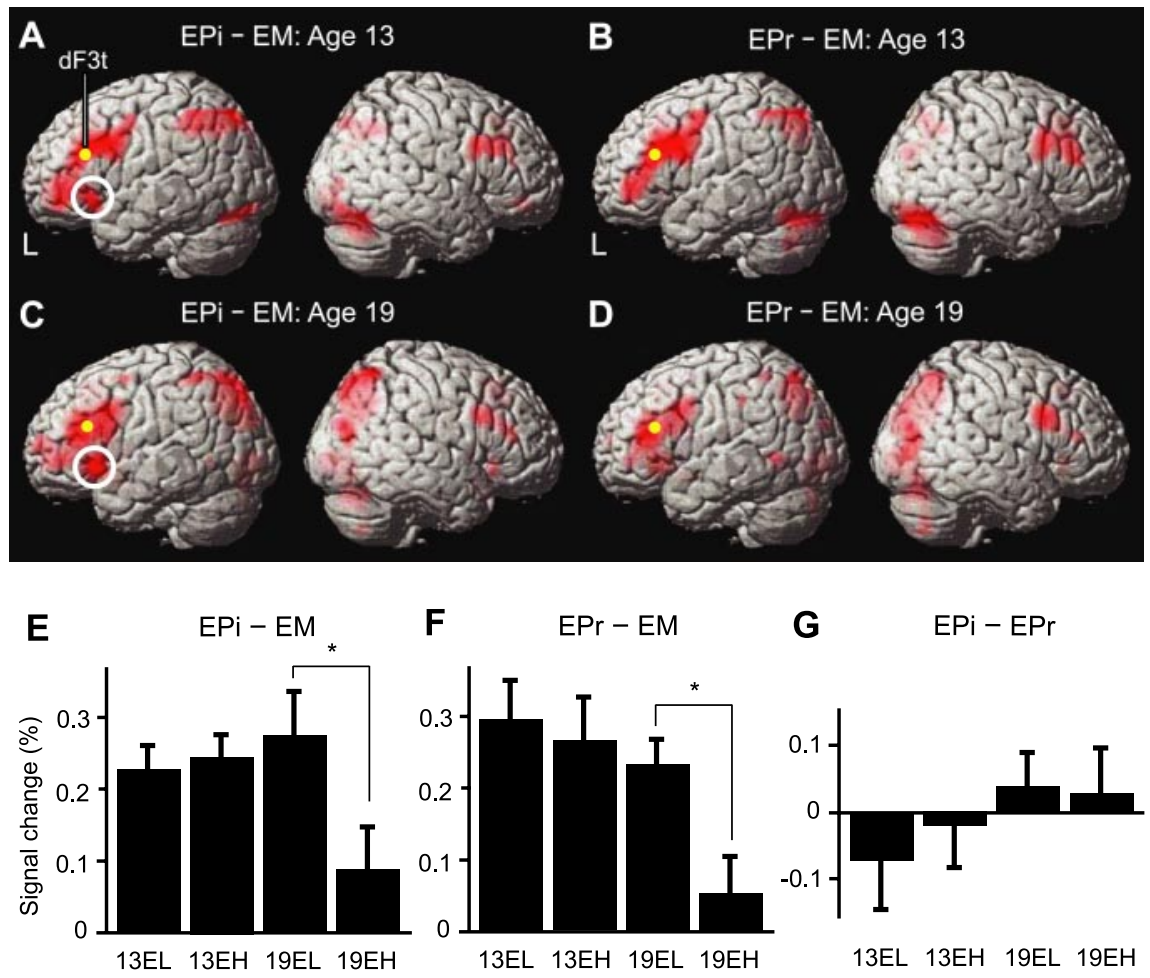

G $\quad E P i-E P r$
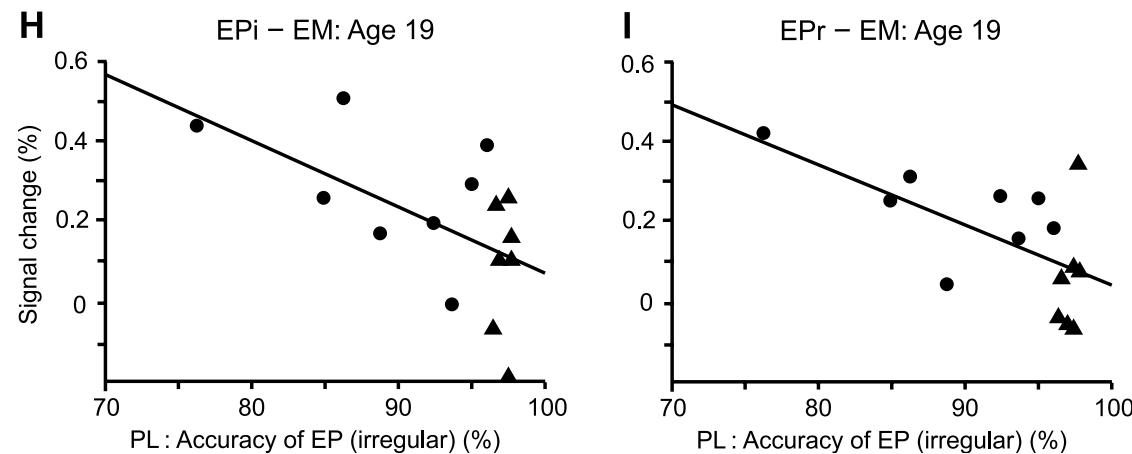

Figure 2. Past-tense task-selective activations in $\mathrm{L} 2$ and the left $\mathrm{dF} 3 \mathrm{t}$ activation modulated by $\mathrm{PL}$. $A-D$, Significant activations in the contrasts of EPi-EM and EPr-EM ( $p<0.05$, corrected for multiple comparisons) for age 13 ( $A$ and $B$, respectively) and those for age 19 ( $C$ and $D$, respectively). The activated regions are projected onto a surface-rendered standard brain (L, left). Note the significant left F3t/F30 activation in EPi-EM for both age 13 and age 19 (white circles in $A$ and $C$ ). E-G, At the left dF3t [( $-51,24$ shown for each subgroup. Asterisks indicate the significant difference in signal changes between the 19EL and 19EH subgroups ( $t$ percentage signal changes in the left $\mathrm{dF} 3 \mathrm{t}(-51,24,24)$ in $\mathrm{EPi}-\mathrm{EM}(H)$ and $\mathrm{EPr}-\mathrm{EM}(I)$ are shown for age 19 . Circles and triangles correspond to the 19EL and 19EH subgroups, respectively. A regression line is shown for each panel.

comparable signal changes between 13EH and 19EL in EPi-EM $(p>0.5)$, despite their significant difference in the performance of EPi (Fig. 1A), indicate that the modulation does not primarily reflect the task performance or difficulty in general. The greater left F3t/F3O activation in EPi-EM for 19EL than for 19EH, together with the nonsignificant activation in EPr-EM for 19EL and $19 \mathrm{EH}$ when the performance of EPr almost reached perfection, suggests that the modulation of the left F3t/F3O activation reflects language task demands for identifying correct past-tense forms.

Left F3t/F3O activation in L1 selectively modulated by age Given the activation modulations of the left IFG (the dF3t and the $\mathrm{F} 3 \mathrm{t} / \mathrm{F} 3 \mathrm{O}$ ) in $\mathrm{L} 2$, we next examined whether the cortical activation could also be modulated in L1, i.e., JP-JM. For the age 13 group, 
Table 1. Activated regions for L2 past-tense verb identification

\begin{tabular}{|c|c|c|c|c|c|c|c|c|c|c|}
\hline Brain regions & $\mathrm{BA}$ & Side & $x$ & $y$ & $Z$ & $Z$ & $x$ & $y$ & $Z$ & Z \\
\hline EPi-EM & & & Age 13 & & & & Age 19 & & & \\
\hline \multirow[t]{3}{*}{ PrCG/F3op/F3t } & $6 / 44 / 45$ & $\mathrm{~L}$ & -48 & 30 & 30 & $>8.0$ & -45 & 30 & 21 & $>8.0$ \\
\hline & & $\mathrm{L}$ & -45 & 9 & 30 & $>8.0$ & -48 & 15 & 30 & $>8.0$ \\
\hline & & $\mathrm{L}$ & -48 & 45 & 3 & $>8.0$ & -45 & 45 & -3 & $>8.0$ \\
\hline F3op/F3t & $44 / 45$ & $\mathrm{R}$ & 54 & 24 & 30 & 6.9 & 54 & 18 & 30 & 7.5 \\
\hline $\mathrm{F} 3 \mathrm{t} / \mathrm{F} 30$ & $45 / 47$ & $\mathrm{~L}$ & -33 & 24 & -3 & $>8.0$ & -33 & 24 & -6 & $>8.0$ \\
\hline $\mathrm{F} 30 / \mathrm{F} 20$ & 47 & $\mathrm{R}$ & 48 & 42 & -9 & 5.6 & & & & \\
\hline Insula & & $\mathrm{R}$ & & & & & 36 & 24 & -6 & 6.7 \\
\hline $\mathrm{F} 2$ & $46 / 9$ & $\mathrm{~L}$ & & & & & -36 & 3 & 57 & 6.8 \\
\hline SMA & 6 & M & 0 & 27 & 45 & $>8.0$ & 0 & 24 & 48 & $>8.0$ \\
\hline Caudate & & $M$ & & & & & -12 & 3 & 9 & 7.2 \\
\hline \multirow[t]{2}{*}{ Parieto-occipital } & 40/39/19/18 & $\mathrm{L}$ & -36 & -48 & 42 & $>8.0$ & -27 & -66 & 39 & $>8.0$ \\
\hline & & $\mathrm{R}$ & 30 & -66 & 39 & 5.9 & 36 & -63 & 60 & 7.6 \\
\hline Calcarine & $17 / 18$ & M & & & & & 12 & -87 & -3 & $>8.0$ \\
\hline \multirow[t]{2}{*}{ Cerebellum } & & $\mathrm{L}$ & -27 & -87 & -15 & 6.0 & -12 & -81 & -33 & 5.3 \\
\hline & & $\mathrm{R}$ & 30 & -69 & -24 & 7.1 & 12 & -81 & -27 & 6.2 \\
\hline EPr-EM & & & Age 13 & & & & Age 19 & & & \\
\hline \multirow[t]{2}{*}{$\mathrm{PrCG} / \mathrm{F} 3 \mathrm{op} / \mathrm{F} 3 \mathrm{t}$} & $6 / 44 / 45$ & $\mathrm{~L}$ & -48 & 30 & 24 & $>8.0$ & -48 & 36 & 18 & $>8.0$ \\
\hline & & $\mathrm{L}$ & -45 & 9 & 30 & $>8.0$ & -42 & 6 & 33 & 7.2 \\
\hline F3op/F3t & $44 / 45$ & $\mathrm{R}$ & 51 & 12 & 30 & 6.8 & 54 & 15 & 30 & $>8.0$ \\
\hline \multirow[t]{2}{*}{ Insula } & & $L$ & & & & & -30 & 27 & 0 & $>8.0$ \\
\hline & & $\mathrm{R}$ & & & & & 33 & 27 & -3 & 5.7 \\
\hline SMA & 6 & M & 3 & 24 & 45 & $>8.0$ & 0 & 24 & 48 & 6.8 \\
\hline Caudate & & M & & & & & -12 & 0 & 9 & 5.0 \\
\hline Middle temporal g. & 21 & $\mathrm{~L}$ & & & & & -57 & -57 & 0 & 6.6 \\
\hline \multirow[t]{2}{*}{ Parieto-occipital } & $40 / 39 / 19 / 18$ & $\mathrm{~L}$ & -42 & -51 & 54 & 7.6 & -27 & -66 & 39 & $>8.0$ \\
\hline & & $\mathrm{R}$ & 30 & -81 & 24 & 6.1 & 33 & -60 & 45 & 7.2 \\
\hline Calcarine & $17 / 18$ & M & & & & & 12 & -87 & -3 & $>8.0$ \\
\hline \multirow[t]{2}{*}{ Cerebellum } & & $\mathrm{L}$ & -45 & -66 & -18 & 7.1 & -9 & -81 & -33 & 6.4 \\
\hline & & $\mathrm{R}$ & 33 & -72 & -21 & $>8.0$ & 9 & -81 & -27 & 6.7 \\
\hline
\end{tabular}

Stereotactic coordinates $(x, y, z)$ in the MNI space are shown for each activation peak of $Z$ values. The threshold is set at corrected $p<0.05$ for voxel level, and clusters smaller than six voxels were removed from the table for brevity. BA Brodmann's area; L, left hemisphere; $R$, right hemisphere; $M$, medial; $g$, gyrus; $F 2$, middle frontal gyrus; SMA, supplementary motor area.

Table 2. Main effects of task and age for $L 2$ past-tense processing

\begin{tabular}{lllllll}
\hline Brain regions & BA & Side & $x$ & $y$ & $z$ & $Z$ \\
\hline EPi - EPr: age $13+$ age 19 & & & & & & \\
\hline F3t/F30 & $45 / 47$ & L & -51 & 24 & -3 & 5.3
\end{tabular}

$($ EPi + EPr)-EM: age 13-age 19

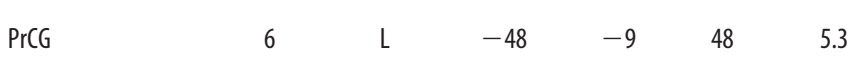

$($ EPi + EPr)-EM: age 19-age 13

\begin{tabular}{lllrrrr}
\hline F3t & 45 & $\mathrm{~L}$ & -39 & 21 & 12 & 4.7 \\
Inferior parietal gyrus & 40 & $\mathrm{~L}$ & -60 & -33 & 39 & $>8.0$ \\
Middle occipital gyrus & $39 / 19$ & $\mathrm{R}$ & 45 & -75 & 18 & $>8.0$ \\
Calcarine & $17 / 18$ & $\mathrm{M}$ & 9 & -78 & -3 & 5.0 \\
\hline
\end{tabular}

Stereotactic coordinates $(x, y, z)$ in the MNI space are shown for each activation peak of $Z$ values. The threshold is set at corrected $p<0.05$ for voxel level, and clusters smaller than six voxels were removed from the table for brevity.

the most prominent activation was localized in the left IFG (cluster size, 624), spanning from the PrCG to the F3op/F3t, including the $\mathrm{dF} 3 \mathrm{t}$ and the F3t/F3O (Fig. 4A, Table 3). It should be noted that the activated regions in the left IFG were similar to those observed in EPi-EM and EPr-EM (Fig. 2A,B). Additional activations were observed in the right $\mathrm{F} 3 \mathrm{O}$ and in the left inferior parietal gyrus. In contrast to the age 13 group, no significant activation was observed for the age 19 group, although the left IFG activation (cluster size, 189) became evident when the threshold was lowered to uncorrected $p<0.001$. In fact, the left dF3t [Fig. $4 A$, yellow dot $(-51,24,24)$ ], which exhibited significant activation for the EP tasks (Fig. $2 A-D$ ), showed comparable activations in JP-JM between the age 13 and age 19 groups $(p>$
0.05) (Fig. 4C). The interaction of task by age, JP-JM: age 13-age 19, revealed a significant activation in the left F3t/F3O [Fig. $4 B$, yellow circle $(-45,21,-3)$ ], the left F3t/F2, and the right F3O. The signal changes in JP -JM at the left F3t/F3O locus were significantly different between the age 13 and age 19 groups $(p<$ 0.005 ) (Fig. $4 D$ ), as well as at the other two loci. It is intriguing that this left F3t/F3O locus matched that found in EPi-EPr: age 19 (Fig. 3A). To confirm that the activation difference in Figure $4 D$ truly reflected the effects of age, we further compared the activation for 13JH and 19JL, whose performances of JP and JM were matched (Fig. 1E,F). The signal changes in JP-JM at the left $\mathrm{F} 3 \mathrm{t} / \mathrm{F} 3 \mathrm{O}$ locus were also significantly different between these subgroups $(p<0.05)$ (Fig. $4 E$ ). Because both the age 13 and age 19 groups were old enough compared with the ideal ages at which to acquire L1 (before 6 years old), their PLs of L1 should be equivalent, and thus the performance-matched 13JH and 19JL subgroups would have comparable task demands for the JP task. Moreover, the JM task served as the control condition for the individual groups, and thus the general developmental changes in cortical activations would have been canceled out in JP-JM. These results suggest that the activation in the left $\mathrm{F} 3 \mathrm{t} / \mathrm{F} 3 \mathrm{O}$ is more sensitive to the comparable language task demands for age 13 than age 19.

\section{Discussion}

In the present study, we demonstrated that the distinct dorsal and ventral regions in the left IFG exhibited language task-selective activations that are indeed modulated by separable factors. The results were striking in three ways. First, the left dF3t activation in EPi-EM and EPr-EM was lower, corresponding to a higher PL 

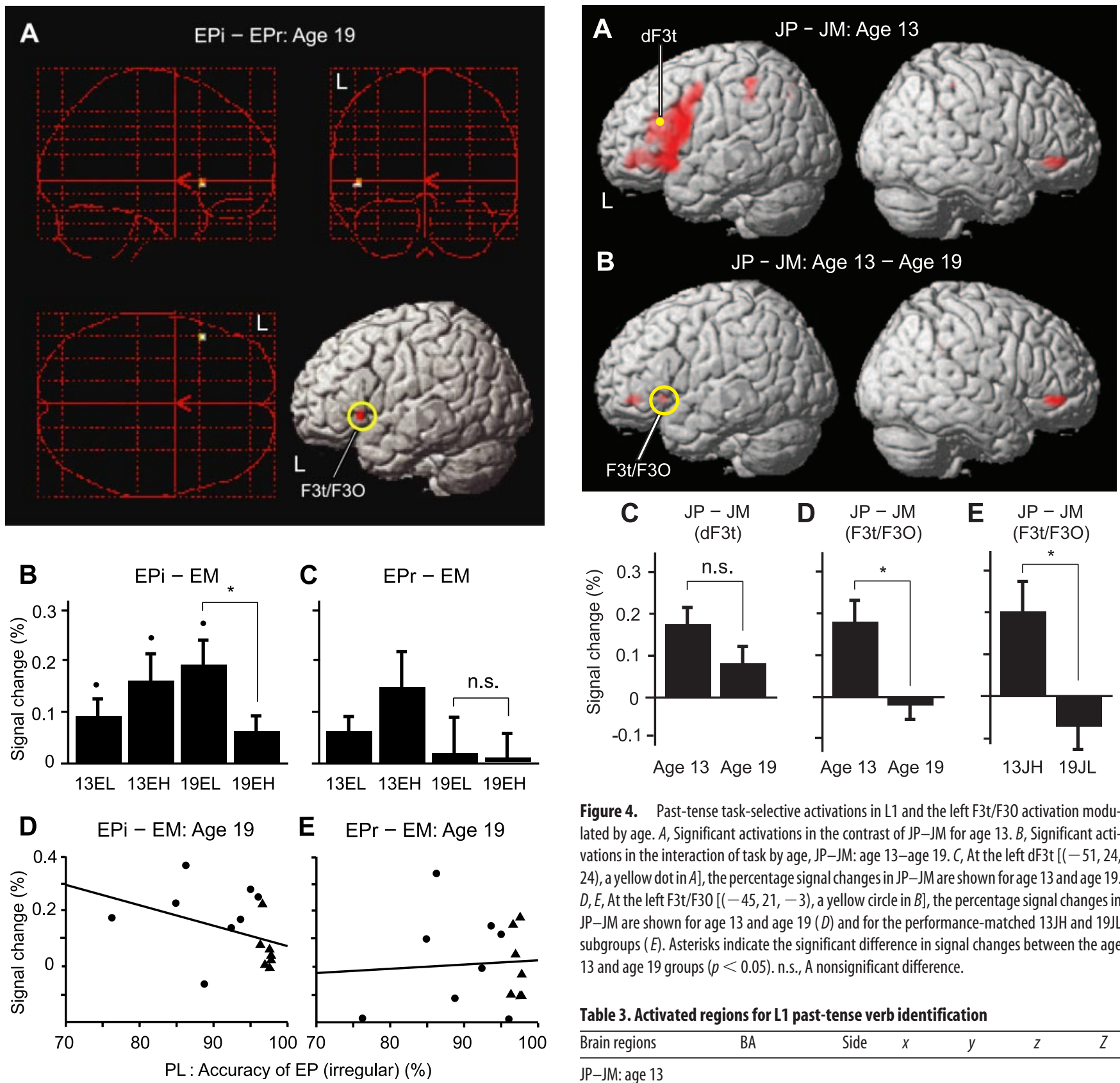

Figure 4. Past-tense task-selective activations in $\mathrm{L} 1$ and the left $\mathrm{F} 3 \mathrm{t} / \mathrm{F} 30$ activation modulated by age. $A$, Significant activations in the contrast of JP-JM for age 13. B, Significant activations in the interaction of task by age, JP-JM: age 13-age 19. C, At the left dF3t [( $-51,24$, 24), a yellow dot in $A$, the percentage signal changes in JP-JM are shown for age 13 and age 19. $D, E$, At the left $\mathrm{F} 3 \mathrm{t} / \mathrm{F} 30[(-45,21,-3)$, a yellow circle in $B]$, the percentage signal changes in JP-JM are shown for age 13 and age 19 (D) and for the performance-matched 13JH and 19J subgroups $(E)$. Asterisks indicate the significant difference in signal changes between the age 13 and age 19 groups $(p<0.05)$. n.s., A nonsignificant difference.

Table 3. Activated regions for $\mathrm{L} 1$ past-tense verb identification

\begin{tabular}{llllrrr}
\hline Brain regions & BA & Side & $x$ & $y$ & \multicolumn{1}{c}{$z$} & $Z$ \\
\hline JP-JM: age 13 & & & & & & \\
\hline PrCG/F3op/F3t/F30 & $6 / 44 / 45 / 47$ & $\mathrm{~L}$ & -48 & 0 & 45 & 6.3 \\
& & $\mathrm{~L}$ & -57 & 9 & 27 & 6.3 \\
& & $\mathrm{~L}$ & -51 & 33 & 15 & 5.5 \\
F30 & $\mathrm{L}$ & -48 & 21 & -6 & 6.6 \\
Inferior parietal gyrus & 47 & $\mathrm{R}$ & 42 & 39 & -6 & 5.8 \\
\hline
\end{tabular}

Figure 3. Left $\mathrm{F} 3 \mathrm{t} / \mathrm{F} 30$ activation in $\mathrm{L} 2$ selectively modulated by language task demands. $A$ A region identified by the contrast EPi-EPr for age 19 was projected in three orthogonal planes (sagittal, coronal, and horizontal from the top left panel) and onto a left surface-rendered standard brain. $B, C$, At the left $F 3 t / F 30[(-48,21,-3)$, a yellow circle in $A]$, the percentage signal changes in EPi-EM $(B)$ and EPr-EM $(C)$ are shown for each subgroup. Dots indicate the significant signal changes ( $t$ test; $p<0.05)$. An asterisk indicates the significant difference in signal changes between the 19EL and 19EH subgroups $(p<0.05) . D, E$, The relationships between $\mathrm{PL}$ and percentage signal changes in the left $\mathrm{F3t} / \mathrm{F3O}(-48,21,-3)$ in EPi-EM (D) and $\mathrm{EPr}-\mathrm{EM}(E)$ are shown for age 19. Circles and triangles correspond to the 19EL and 19EH subgroups, respectively. A regression line is shown for each panel.

for age 19, suggesting that PL plays a major role in the left dF3t activation during $\mathrm{L} 2$ acquisition. Second, the greater left F3t/F3O activation in EPi-EM for 19EL than for 19EH, together with the nonsignificant activation in EPr-EM for 19EL and 19EH when the performance of EPr almost reached perfection, suggests that the modulation of the left $\mathrm{F} 3 \mathrm{t} / \mathrm{F} 3 \mathrm{O}$ activation reflects language task demands for identifying correct past-tense forms. Third, in

Stereotactic coordinates $(x, y, z)$ in the MNI space are shown for each activation peak of $Z$ values. The threshold is set at corrected $p<0.05$ for voxel level, and clusters smaller than six voxels were removed from the table for brevity.

JP-JM, the left F3t/F3O activation for age 13 was significantly greater than that for age 19, even if the performances of JP and JM were matched between the 13JH and 19JL subgroups. Together, the present results provide converging evidence that the left $\mathrm{dF} 3 \mathrm{t}$ and the left $\mathrm{F} 3 \mathrm{t} / \mathrm{F} 3 \mathrm{O}$ activations are differentially modulated by age, PL, and language task demands. These results further suggest that the cortical plasticity for L2 acquisition specifically involves the left IFG, observable even in adolescents.

We reported previously that the left $\mathrm{F} 3 \mathrm{t} / \mathrm{F} 3 \mathrm{O}[(-54,33,-3)$ and $(-54,27,-6)$ according to Homae et al., 2002], as well as the 
left F3op/F3t $[(-57,18,0)$ and $(-57,9,6)$ according to Embick et al., 2000; Suzuki and Sakai, 2003], was selectively activated when adult subjects performed sentence tasks in L1, which located closely to the left F3t/F3O $(-48,21,-3)$ in the present study. Here we reported the age-related modulation of the activation of this region even after fully acquiring L1 (Fig. 4D,E). We suspect that the weaker activation in JP-JM for age 19 was attributable to the higher stringency of this task contrast compared with that of the previous studies and that the higher task-selective sensitivity for age 13 than age 19 resulted in enhanced activation for the same contrast. Schlaggar et al. (2002) reported age-related activation differences independent of language tasks, which may reflect general cognitive factors. Indeed, previous neuroimaging studies have reported a repetition priming effect in which the repeated presentation of visual objects resulted in an activation decrease in broad regions, including the frontal and occipital cortices (Schacter and Buckner, 1998; Van Turennout et al., 2000; Dehaene et al., 2001; Soon et al., 2003). Because the order of tasks was counterbalanced across subjects in the present study, the repetition or familiarization effects on activation, if any, cannot explain the results of JP-JM (Fig. 4). Recent fMRI studies also indicated that the left orbitofrontal cortex [e.g., BA 45/47 ( -45 , $23,-4)$ according to Kesler-West et al., 2001] is related to emotional and social experience (Sprengelmeyer et al., 1998; KeslerWest et al., 2001; Berthoz et al., 2002). However, these factors, if any, would have been canceled out in JP-JM, as well as in EP-EM, for the individual groups in the present study. On the other hand, it has been established recently that the left IFG is selectively involved in grammatical processing (Stromswold et al., 1996; Dapretto and Bookheimer, 1999; Kang et al., 1999; Embick et al., 2000; Friederici et al., 2000; Sakai et al., 2002; Suzuki and Sakai, 2003), independent of general cognitive factors such as task difficulty and short-term memory (Hashimoto and Sakai, 2002), and of unreal language rule usage (Musso et al., 2003). The present results suggest that the modulation of the left F3t/F3O activation is related to the sensitivity to specific language task demands rather than associated with cognitive development in general (Piattelli-Palmarini, 1980; Karmiloff and KarmiloffSmith, 2001).

The developmental changes in regional cerebral blood flow and cerebral metabolic rates are known to manifest initially as an increase, and later, after age of $\sim 9$, as a decrease, reaching adult levels between the ages of 15 and 19 (Chugani et al., 1987; Chiron et al., 1992). Therefore, physiological differences between children and adults might affect the acquisition, analysis, and interpretation of fMRI data in group analyses. However, recent neuroimaging studies have clarified that both anatomical and functional differences between children (aged 7-8) and adults have no significant effects on the fMRI data (Burgund et al., 2002; Kang et al., 2003). In the present study, the EM and JM tasks served as the control condition for the individual groups and thus the general developmental changes in cortical activations would have been canceled out in EP-EM and JP-JM. Therefore, the present results indicate that language-specific factors have stronger modulation effects on the left prefrontal regions, which can be further differentiated within the left IFG.

Combining the findings of our previous study (Sakai et al., 2004) and those of the present one, we clarified that the left dF3t activation increases with PL improvements at the earlier stage of $\mathrm{L} 2$ acquisition and that it is lower when a higher proficiency in L2 is attained (Fig. 2E,F). This latter result is consistent with a previous fMRI study reporting weaker left IFG activation for bilingual subjects with higher PLs during a semantic decision task
(Chee et al., 2001). Regarding the initial increase in activation, consistent results have been reported recently showing that activation in the left F3t increases when subjects first learn the grammatical rules of L2 (Musso et al., 2003). On the other hand, another fMRI study reported that the left IFG is more activated by making grammatical judgments in L2 than in L1, but that it occurred independent of PLs in L2 (Wartenburger et al., 2003). However, the present results indicate that the left dF3t may show higher, lower, or comparable activation, depending on which stages of L2 acquisition are compared. Wartenburger et al. (2003) also reported that the left IFG activation is greater for subjects at a later AOA than for those at an earlier AOA, even when their high PLs were matched. Here, it should be noted that the subject groups' mean intervals of exposure to two languages in their study differed by 16 years, and thus the differences between those groups might have disappeared if the degree of exposure had been equated. Additional research is necessary to determine whether the left IFG activation depends on exposure to the language at a particular stage of the developing brain.

Finally, we posed one remaining question about the nature of language task demands that elicited more enhanced activation in the left F3t/F3O for age 13 than for age 19 (Fig. 4), which may also modulate the activation of the same region before completely mastering past-tense regularity in L2 (Fig. 3). A recent fMRI study with lexico-semantic tasks proposed that the left F3t/F3O is related to controlled semantic retrieval (Wagner et al., 2001). The local maximum of the activated region in the study by Wagner et al. (2001) was actually in F3O. Another fMRI study suggested that the left F3t/F3O is related to controlled retrieval of nonsemantic information as well as semantic attributes at the lexical level (Gold and Buckner, 2002). It remains possible that the activation can be modulated by such retrieval of nonsemantic information, but the present study suggests that it may specifically reflect the demands of retrieving past-tense forms that the EP tasks required. This possibility is consistent with the greater signal change in EPi-EM for 19EL than for 19EH (Fig. 3B), as well as the nonsignificant signal change in EPr-EM for 19EL and 19EH (Fig. $3 C$ ) when the performance of EPr almost reached perfection (Fig. $1 \mathrm{~B})$. Another possibility for language task demands would be syntactic demands for past tense processing. If we assume that the syntactic process is involved in making not only regular but irregular past-tense forms, the greater signal change in EPi- EM for 19EL than for 19EH (Fig. 3B), as well as the nonsignificant signal change in EPr-EM for 19EL and 19EH (Fig. 3C), can also be explained by the difference in syntactic demands. We prefer this possibility, because it is supported by accumulating evidence of the syntactic specialization in the left IFG, as discussed above. Taking these results together, we reached the conclusion that the left IFG subserves language-specific functions that are critically required when mastering any language.

\section{References}

Berthoz S, Armony JL, Blair RJR, Dolan RJ (2002) An fMRI study of intentional and unintentional (embarrassing) violations of social norms. Brain 125:1696-1708.

Burgund ED, Kang HC, Kelly JE, Buckner RL, Snyder AZ, Petersen SE, Schlaggar BL (2002) The feasibility of a common stereotactic space for children and adults in fMRI studies of development. NeuroImage 17:184-200.

Chee MWL, Tan EWL, Thiel T (1999) Mandarin and English single word processing studied with functional magnetic resonance imaging. J Neurosci 19:3050-3056.

Chee MWL, Hon N, Lee HL, Soon CS (2001) Relative language proficiency modulates BOLD signal change when bilinguals perform semantic judgments. NeuroImage 13:1155-1163. 
Chiron C, Raynaud C, Mazière B, Zilbovicius M, Laflamme L, Masure M-C, Dulac O, Bourguignon M, Syrota A (1992) Changes in regional cerebral blood flow during brain maturation in children and adolescents. J Nucl Med 33:696-703.

Chugani HT, Phelps ME, Mazziotta JC (1987) Positron emission tomography study of human brain functional development. Ann Neurol 22:487-497.

Dapretto M, Bookheimer SY (1999) Form and content: dissociating syntax and semantics in sentence comprehension. Neuron 24:427-432.

Dehaene S, Dupoux E, Mehler J, Cohen L, Paulesu E, Perani D, Van de Moortele P-F, Lehéricy S, Le Bihan D (1997) Anatomical variability in the cortical representation of first and second language. NeuroReport 8:3809-3815.

Dehaene S, Naccache L, Cohen L, Le Bihan D, Mangin JF, Poline JB, Rivière D (2001) Cerebral mechanisms of word masking and unconscious repetition priming. Nat Neurosci 4:752-758.

Embick D, Marantz A, Miyashita Y, O’Neil W, Sakai KL (2000) A syntactic specialization for Broca's area. Proc Natl Acad Sci USA 97:6150-6154.

Friederici AD, Opitz B, von Cramon DY (2000) Segregating semantic and syntactic aspects of processing in the human brain: an fMRI investigation of different word types. Cereb Cortex 10:698-705.

Gold BT, Buckner RL (2002) Common prefrontal regions coactivate with dissociable posterior regions during controlled semantic and phonological tasks. Neuron 35:803-812.

Hashimoto R, Sakai KL (2002) Specialization in the left prefrontal cortex for sentence comprehension. Neuron 35:589-597.

Homae F, Hashimoto R, Nakajima K, Miyashita Y, Sakai KL (2002) From perception to sentence comprehension: the convergence of auditory and visual information of language in the left inferior frontal cortex. NeuroImage 16:883-900.

Johnson JS, Newport EL (1989) Critical period effects in second language learning: the influence of maturational state on the acquisition of English as a second language. Cognit Psychol 21:60-99.

Kang AM, Constable RT, Gore JC, Avrutin S (1999) An event-related fMRI study of implicit phrase-level syntactic and semantic processing. NeuroImage 10:555-561.

Kang HC, Burgund ED, Lugar HM, Petersen SE, Schlaggar BL (2003) Comparison of functional activation foci in children and adults using a common stereotactic space. NeuroImage 19:16-28.

Karmiloff K, Karmiloff-Smith A (2001) Pathways to language-from fetus to adolescent. Cambridge, MA: Harvard UP.

Kesler-West ML, Andersen AH, Smith CD, Avison MJ, Davis CE, Kryscio RJ, Blonder LX (2001) Neural substrates of facial emotion processing using fMRI. Brain Res Cogn Brain Res 11:213-226.

Kim KHS, Relkin NR, Lee KM, Hirsch J (1997) Distinct cortical areas associated with native and second languages. Nature 388:171-174.
Lenneberg EH (1967) Biological foundations of language. New York: Wiley. Musso M, Moro A, Glauche V, Rijntjes M, Reichenbach J, Büchel C, Weiller C (2003) Broca's area and the language instinct. Nat Neurosci 6:774-781.

Perani D, Paulesu E, Galles NS, Dupoux E, Dehaene S, Bettinardi V, Cappa SF, Fazio F, Mehler J (1998) The bilingual brain — proficiency and age of acquisition of the second language. Brain 121:1841-1852.

Perani D, Abutalebi J, Paulesu E, Brambati S, Scifo P, Cappa SF, Fazio F (2003) The role of age of acquisition and language usage in early, highproficient bilinguals: an fMRI study during verbal fluency. Hum Brain Mapp 19:170-182.

Piattelli-Palmarini M (1980) Language and learning. The debate between Jean Piaget and Noam Chomsky. Cambridge, MA: Harvard UP.

Sakai KL, Noguchi Y, Takeuchi T, Watanabe E (2002) Selective priming of syntactic processing by event-related transcranial magnetic stimulation of Broca's area. Neuron 35:1177-1182.

Sakai KL, Miura K, Narafu N, Muraishi M (2004) Correlated functional changes of the prefrontal cortex in twins induced by classroom education of second language. Cereb Cortex 14:1233-1239.

Schacter DL, Buckner RL (1998) Priming and the brain. Neuron 20: 185-195.

Schlaggar BL, Brown TT, Lugar HM, Visscher KM, Miezin FM, Petersen SE (2002) Functional neuroanatomical differences between adults and school-age children in the processing of single words. Science 296:1476-1479.

Soon CS, Venkatraman V, Chee MWL (2003) Stimulus repetition and hemodynamic response refractoriness in event-related fMRI. Hum Brain Mapp 20:1-12.

Sprengelmeyer R, Rausch M, Eysel UT, Przuntek H (1998) Neural structures associated with recognition of facial expressions of basic emotions. Proc R Soc Lond B Biol Sci 265:1927-1931.

Stromswold K, Caplan D, Alpert N, Rauch S (1996) Localization of syntactic comprehension by positron emission tomography. Brain Lang 52: $452-473$.

Suzuki K, Sakai KL (2003) An event-related fMRI study of explicit syntactic processing of normal/anomalous sentences in contrast to implicit syntactic processing. Cereb Cortex 13:517-526.

Van Turennout M, Ellmore T, Martin A (2000) Long-lasting cortical plasticity in the object naming system. Nat Neurosci 3:1329-1334.

Wagner AD, Paré-Blagoev EJ, Clark J, Poldrack RA (2001) Recovering meaning: left prefrontal cortex guides controlled semantic retrieval. Neuron 31:329-338.

Wartenburger I, Heekeren HR, Abutalebi J, Cappa SF, Villringer A, Perani D (2003) Early setting of grammatical processing in the bilingual brain. Neuron 37:159-170. 